\title{
Kinetic Temperature and Electron Density Measurement in an Inductively Coupled Plasma Torch using Degenerate Four-Wave Mixing
}

\author{
Julia Schafer, Wendy Lyons and William G. Tong* \\ Department of Chemistry, San Diego State University, San Diego, California 92182, USA \\ Paul M. Danehy \\ Advanced Sensing and Optical Measurement Branch, NASA Langley Research Center, Hampton \\ VA 23681-2199, USA
}

\begin{abstract}
Laser wave mixing is presented as an effective technique for spatially resolved kinetic temperature measurements in an atmospheric-pressure radio-frequency inductively-coupled plasma. Measurements are performed in a $1 \mathrm{~kW}, 27 \mathrm{MHz}$ RF plasma using a continuous-wave, tunable 811.5-nm diode laser to excite the $4 \mathrm{~s}^{3} \mathrm{P}_{2} \rightarrow 4 \mathrm{p}^{3} \mathrm{D}_{3}$ argon transition. Kinetic temperature measurements are made at five radial steps from the center of the torch and at four different torch heights. The kinetic temperature is determined by measuring simultaneously the line shape of the sub-Doppler backward phase-conjugate degenerate four-wave mixing and the Dopplerbroadened forward-scattering degenerate four-wave mixing. The temperature measurements result in a range of 3,500 to $14,000 \mathrm{~K} \pm 150 \mathrm{~K}$. Electron densities measured range from $6.1( \pm$ $0.3) \times 10^{15} \mathrm{~cm}^{-3}$ to $10.1( \pm 0.3) \times 10^{15} \mathrm{~cm}^{-3}$. The experimental spectra are analyzed using a perturbative treatment of the backward phase-conjugate and forward-geometry wave-mixing theory. Stark width is determined from the collisional broadening measured in the phaseconjugate geometry. Electron density measurements are made based on the Stark width. The kinetic temperature of the plasma was found to be more than halved by adding deionized water through the nebulizer.
\end{abstract}




\section{Introduction}

Radio-frequency inductively coupled plasma (RF-ICP) torches have a variety of uses in materials processing, analytical chemistry, non-equilibrium physics, and radiative heat transfer studies. The temperature of the gases in the torch is of critical interest to the use and modeling of these plasmas. Several different temperature measurement methods have previously been used including emission spectroscopy for the highly-luminous environment. [1, 2]. However, emission spectroscopy is a line-of-sight measurement, and thus, not spatially-precise. If the plasma is axisymmetric, this limitation can be overcome by invoking Abel inversion, although it is non-trivial and is susceptible to error propagation. Emission spectroscopy frequently uses the relative intensities of several different spectral emission lines to determine an electronic temperature. These relative intensities describe the mean distribution of the electron populations in the excited electronic states of an atom such as argon. Broadening of spectral lines, such as hydrogen, can yield information about the plasma. For example, the kinetic temperature from Doppler broadening and the Stark broadening can be used to infer the electron number density from hydrogen atoms.

Linear absorption techniques have also been applied to atmospheric plasma torches. Both Baer [3] and De Regt [4] used tunable diode lasers for line-of-sight absorption measurements in atmospheric pressure plasma. In 2002, Wang et al. [5] applied cavity ring down spectroscopy on argon in a plasma torch. However, absorption and cavity ring down spectroscopic methods are still based on line-of-sight measurements.

An effective technique that has been used to measure temperature in plasmas is laserinduced fluorescence (LIF) [6]. LIF has the advantage over absorption or emission methods 
since it is a spatially-precise technique with the measurement location defined by the intersection of the laser beam and the detection system. Thus, the assumption of axisymmetry is not required. However, laser-induced fluorescence is scattered isotropically. Using low-power diode lasers in such a luminous environment requires very long duration measurements so that the weak LIF signal can be discriminated from the strong ambient emission. Similarly, De Regt et al. [7] compared Thomson and Raleigh scatterings using a $100 \mathrm{MHz}$ argon ICP. However, both of these processes are relatively weak. Therefore, in luminous environments, either very long measurement times or high-power lasers are required previously to obtain good signal-tonoise ratios.

One method that offers some advantages over current techniques is degenerate four-wave mixing (DFWM), a nonlinear optical method used effectively in combustion research for temperature and species density measurements [8]. It is a spatially precise technique with the measurement location defined by the overlap volume of three input laser beams. Wave mixing offers two main advantages over laser-induced fluorescence. First, the wave-mixing signal is relatively insensitive to quenching collisions [9], and hence, the signal relates more directly to species density (or state population in the case that an excited state is probed) than in LIF. Second, the wave-mixing signal is a coherent laser-like beam, and hence, spatial filtering can be used very effectively to improve the signal-to-noise ratio. Green et al. [10] used pulsed DFWM to probe a RF-ICP torch and measured the vibrational temperature of $\mathrm{CH}$ and the $\mathrm{CH}$ concentration of multiple $\mathrm{CH}$ vibrational levels. Musiol et al. [11] made assumptions about the local thermodynamic state of the plasma to obtain an approximate electronic temperature of argon, based only on the intensity of a single spectroscopic line. 
In this study, we use DFWM to measure the kinetic temperature of argon atoms by simultaneously detecting the forward- and backward-scattering DFWM spectra. Most previous DFWM measurements used the so-called backward-scattering phase-matching geometry (i.e., phase-conjugate geometry) [11]. More recently, the forward geometry has been investigated and different groups reported that forward DFWM spectra are Doppler broadened whereas backward DFWM spectra yield sub-Doppler or Doppler-free in the limit of small beam crossing angles [12]. Danehy et al. $[13,14]$ obtained forward-geometry spectra of nitric oxide in a gas cell, and subsequently obtained backward-geometry spectra under the same nominal conditions with a different optical setup. By sequentially fitting both spectra using perturbation theory [15], Danehy et al. [13] were able to determine both the Doppler broadening and the homogeneous broadening. From these broadening parameters, the gas temperature and pressure, were determined using a well-controlled gas cell. These results validated DFWM for quantitative measurement of temperature and pressure.

The present study substantially extends this capability in three ways. First, the technique is applied to a continuously flowing popular analytical atomizer (inductively coupled plasma using argon). Second, a novel beam alignment is used to obtain the forward- and backwardgeometry spectra simultaneously, as opposed to using separate optical setups. Third, low-power, high-resolution, tunable diode lasers are used. The low-power laser is split into multiple beams and directed through a small sample volume in the plasma. The input beams are tuned to an argon line at $811.754 \mathrm{~nm}$. Both forward- and backward-geometry DFWM signals are detected simultaneously using two different photodetectors. The spectra are subsequently processed to yield temperature and homogeneous broadening levels. Since the homogeneous broadening is dominated by Stark broadening under these conditions [3], the electron number density can be 
inferred from the homogenous broadening. Our dual-DFWM technique is presented as an effective method for mapping the temperature and electron density in a RF-ICP torch at typical operating conditions.

\section{Theory}

In 1978, Abrams and Lind presented a model for DFWM in an absorbing, homogeneously broadened two-level media [16]. However, this model does not take into account atomic motion. To address the effects of atomic motion, Nilsen and Yariv [15] presented a perturbative framework for predicting DFWM spectra and intensities for any phasematching geometry. Abrams et al. [17] simplified these expressions for the case of phaseconjugate phase-matched wave mixing. In 1995, Danehy evaluated the perturbation-theory solution for the case of the forward phase-matching geometry [18]. The line shapes for DFWM in both the phase-conjugate and forward setups were derived from the general formulation of Nilsen and Yariv [15]. The resulting expressions assumed that the three input beams are collinear. This is a valid assumption provided that $\theta<2 \gamma / \mathrm{kv}_{\mathrm{o}}\left(\gamma=\right.$ natural linewidth $/ \mathrm{kv} v_{0}=$ Doppler width). This analysis includes (1) inter-molecular collisions, which lead to pressure (homogeneous) broadening, and (2) molecular motion, which results in Doppler (inhomogeneous) broadening.

The phase-conjugate geometry is often called the backward phase-matching geometry. In this experiment, we use a non-planar counter-propagating beam geometry (described below). In the limit of small $\theta$, where $\theta<2 \gamma / \mathrm{kv}_{0}$, then the normalized wave vectors are:

$$
\hat{n}_{f}=\hat{n}_{p}=-\hat{n}_{b}=-\hat{n}_{s}
$$


The line shape for the backward geometry can be described as [18]:

$$
S_{b k w d}(\Delta, \Psi, \xi) \propto\left|\kappa_{s, b k w d, b p}+\kappa_{s, b k w d, f p}\right|^{2}
$$

where $\kappa_{S}\left(\vec{v}_{N}\right)$ is velocity-dependent nonlinear coupling constant, which can be derived for the backward DFWM as:

$$
\begin{aligned}
& \kappa_{s, b k w d, b p, D F W M} \propto \frac{i \pi}{k}\left[\frac{-\frac{d}{d z} w_{f}\left(\frac{-\Delta+i \gamma_{12}}{k v_{0}}\right)}{k v_{0}\left(2 \Delta-2 i \gamma_{12}+i \Gamma_{0}\right)}-\frac{2 w_{f}\left(\frac{-\Delta+i \gamma_{12}}{k v_{0}}\right)}{\left(2 \Delta-2 i \gamma_{12}+i \Gamma_{0}\right)^{2}}\right] \\
& +\frac{i \pi}{2 k \Delta}\left[\frac{w_{f}\left(\frac{-\Delta+i \gamma_{12}}{k v_{0}}\right)}{2 \Delta-2 i \gamma_{12}+i \Gamma_{0}}-\frac{w_{f}\left(\frac{\Delta+i \gamma_{12}}{k v_{0}}\right)}{-2 \Delta-2 i \gamma_{12}+i \Gamma_{0}}\right] \\
& +\frac{2 i \pi w_{f}\left(\frac{i \Gamma_{0}}{2 k v_{0}}\right)}{k\left(2 \Delta-2 i \gamma_{12}+i \Gamma_{0}\right)}\left[\frac{1}{2 \Delta-2 i \gamma_{12}+i \Gamma_{0}}-\frac{1}{2 \Delta+2 i \gamma_{12}+i \Gamma_{0}}\right] \\
& \kappa_{s, b k w d, f p, D F W M} \propto \frac{\pi}{k \Gamma_{0}}\left[\frac{w_{f}\left(\frac{-\Delta+i \gamma_{12}}{k v_{0}}\right)}{-\Delta+i \gamma_{12}}-\frac{\left(w_{f}\left(\frac{-\Delta+i \gamma_{12}}{k v_{0}}\right)-w_{f}\left(\frac{\Delta+i \gamma_{12}}{k v_{0}}\right)\right)}{2 \Delta}\right]
\end{aligned}
$$

where $a=\gamma_{12} / \Gamma_{o}, \alpha_{o}$ is the line-center absorption coefficient, $\gamma_{12}$ is the homogeneous broadening, $\Gamma_{0}$ is the population decay rate, $\mathrm{kv}_{0}$ is the Doppler width, $\Delta=\omega_{o}-\omega_{f}$, and

$$
w_{f}(z)=\frac{i}{\pi} \int_{-\infty}^{\infty} \frac{e^{-t^{2}}}{z-t} d t
$$


In the forward geometry, if $\theta<2 \gamma / \mathrm{kv}_{0}$, then all four beams have effectively identical normalized wave vectors:

$$
\hat{n}_{f}=\hat{n}_{p}=\hat{n}_{b}=\hat{n}_{s}
$$

We also use the out-of-plane forward geometry. The line shape for the forward geometry [18] is:

$$
S_{f w d}(\Delta, \Psi, \xi) \propto\left|\kappa_{s, f w d}\right|^{2}
$$

where $\kappa_{S}\left(\vec{v}_{N}\right)$ is the velocity-dependent nonlinear coupling constant. The nonlinear coupling constant derived for the forward DFWM is as follows:

$$
\kappa_{s, f w d, D F W M} \propto \frac{-2 \pi}{k^{2} \Gamma_{o} v_{o}} \frac{d}{d z}\left(w_{f}\left(\frac{-\Delta+i \gamma_{12}}{k v_{o}}\right)\right)+\frac{\pi}{i k \Gamma_{o} \lambda_{12}}\left(w_{f}\left(\frac{-\Delta+i \gamma_{12}}{k v_{o}}\right)+w_{f}\left(\frac{\Delta+i \gamma_{12}}{k v_{o}}\right)\right)
$$

Unlike the backward phase-conjugate geometry, the forward geometry spectrum is Doppler broadened significantly, even at small-crossing angles. Isolating the Doppler broadening of the argon spectral line allows one to measure the kinetic temperature.

Since the backward-geometry line width is weakly dependent on the temperature, the analysis method is iterative. An initial guess at temperature, $T_{1}$, is made and the phase-conjugate spectrum is fitted using backward-geometry perturbation theory to determine a first estimate of the homogeneous broadening, $\Delta \mathrm{v}_{\text {homog. }} . T_{2}$ is then determined using $\Delta v_{\text {homog }}$ by fitting the forward-geometry line shape with perturbation theory. This provides an improved estimate of the temperature. If $T_{1}$ equals $T_{2}$ to some acceptable accuracy, then $T_{2}$ is the final measured temperature. If $T_{1}$ does not equal $T_{2}$, then this improved value of the temperature can be used to 
determine an improved value of $\Delta y_{\text {homog. }}$. One can continue to iterate the temperature to get the desired precision for the final temperature.

In order to calculate the electron density, one can examine the homogenous broadening effects. Many processes contribute to the homogenous broadening of a spectral line such as van de Waal, resonance and Stark broadening.

Collisions of the absorbing particle with neutral particles cause collisional broadening referred to as van de Waal broadening. It has been shown that van der Waal broadening in argon contributes $\approx 0.1 \%$ of the total collision width, and can therefore be neglected [19].

When the colliding particles are of the same species, another mechanism called resonance broadening occurs. These interactions are significant for transitions that are radiatively coupled to the ground state. However, transitions that originate from the argon metastable state, such as the $4 \mathrm{~s}^{3} \mathrm{P}_{2}$ probed in this work, are not significantly resonance broadened, because of the relatively small oscillator strengths to the ground state [19].

Stark broadening refers to collisions with charged particles or particles with a strong permanent electric dipole moment. This effect can be understood by considering the effect of an external field on the energy levels of an atomic system and the resulting influence on the spectral line. An electric dipole $\alpha \mathrm{F}$, where $\alpha$ is the atomic polarizabiltiy, is induced by the application of an external electric field F. The interaction between the induced-dipole moment and the electric field shifts the atomic energy levels by an amount proportional to the interaction energy given by $\alpha F^{2}$. Hence, the Stark shift has a quadratic dependence on the electric field strength. The 
influence of many colliding partners causes the Stark effect to result in an overall broadening of the spectral line.

Tabulated theoretical Stark parameters relate the broadening and shift of spectral lines to electron density and temperature. The Stark parameters for the $4 \mathrm{~s}^{3} \mathrm{P}_{2} \rightarrow 4 \mathrm{p}^{3} \mathrm{D}_{3}$ argon transition can be found in Griem [20]. For predominantly singly-ionized plasmas, the full width half maximum (FWHM) due to the quadratic Stark effect, $\mathrm{w}_{\text {th }}(\mathrm{A})$, is given by [19]:

$$
\mathrm{w}_{\text {th }}=2\left[1+1.75 \times 10^{-4} \mathrm{n}_{\mathrm{e}}^{1 / 4} \alpha\left(1-0.068 \mathrm{n}_{\mathrm{e}}^{1 / 6} \mathrm{~T}_{\mathrm{e}}^{-1 / 2}\right)\right] 10^{-16} \mathrm{w} \mathrm{n}_{\mathrm{e}}
$$

where $w$ is the electron impact parameter, $\alpha$ is the ion-broadening parameter, $\mathrm{n}_{\mathrm{e}}$ is the electron density, and $T_{e}$ is the electron temperature. Non-equilibrium calculations suggest that the gas and electron kinetic temperatures should be identical [3]. Hence, all species are assumed to be at the atomic kinetic temperature. The measured temperature and collisional width are matched to Eq. 9 and solved for electron density.

\section{Experimental}

A $27 \mathrm{MHz}$ radio-frequency (RF) inductively coupled plasma (Varian, Inc., custom unit) operating at $1 \mathrm{~kW}$ incident power is used in this work. The plasma torch, as shown in Figure 1, consists of a series of three concentric quartz tubes that carry argon at different flow velocities through the RF coil region. The outer argon flow (typically 5-15 L/min) serves to sustain the plasma and to carry the heat of the plasma away from the quartz glass walls of the torch. The intermediate tube gas flow (typically $0.5-2.0 \mathrm{~L} / \mathrm{min}$ ) serves to lift the plasma a few millimeters from the RF coils. Upon igniting the torch, the intermediate or "auxiliary" flow is made electrically conductive by a Tesla spark before it passes through the RF coil. A RF power of 
approximately $0.5-2 \mathrm{~kW}$ is transferred using a coil wrapped around the torch. The RF field inductively heats and subsequently ionizes the formed plasma to temperatures exceeding $5000 \mathrm{~K}$. The entire process of forming and stabilizing an ICP takes several milliseconds.

The centrally located gas flow or nebulizer flow (typically $0.3-1.5 \mathrm{~L} / \mathrm{min}$ ) is used to introduce analyte into the existing plasma. While passing through the induction region, the aerosol is desolvated, dissociated, atomized and excited. Only deionized water is used in the present experiment.

The optical setup, as shown in Figure 2, is a combination of the backward phaseconjugate geometry and the forward phase-matched geometry. The diode laser system used consists of a low-noise current source (ILX Lightwave Corp., model LDX-3320), thermoelectric temperature controller (ILX Lightwave Corp., model LDX-5525), and laser diode mount (ILX Lightwave Corp., model LDM-5910B). The laser diode (Optima Precision, model Sharp LT016MD) is operated at $36.6^{\circ} \mathrm{C}$ and wavelength tuned by changing the current. The diode has an average power of $25 \mathrm{~mW}$. The diode wavelength is verified by a high-resolution wavemeter (Burleigh Instruments, model WA-1500).

The laser output beam is split into three input beams. A $50 / 50 \% \mathrm{R} / \mathrm{T}$ beam splitter is used to create the backward input beam, $\mathrm{E}_{\mathrm{b}}$. A second 70/30\% R/T beam splitter is used to produce the probe $E_{p}$ and the forward $E_{f}$ input beams. The three beams are easily aligned using our custom-made alignment templates. The beams are left collimated as they pass through the torch (i.e., no focusing lens is used). The beam diameter is $0.8 \mathrm{~mm}$ at the torch and the crossing angle between the input beams is $1.25 \mathrm{deg}$. When positioned at the widest portion of the ICP torch (15 mm wide), the diamond-shaped probe volume created by the overlapping input beams 
inside the torch is estimated to be $13 \mu \mathrm{L}$. Hence, our 2D and 3D spatial resolution levels of 8 $\mathrm{mm}$ and $13 \mu \mathrm{L}$ respectively are comparable or better than those of other methods.

In order to arrange the backward and forward setups simultaneously, the backward pump is tilted at a slight angle to create the backward phase-conjugate geometry first. The backward pump, $\mathrm{E}_{\mathrm{b}}$, is then reflected back on itself using the alignment template to form the second pump beam for the forward phase-matched geometry. Care must be taken to use spatial filters in order to minimize light retracing back into the laser and potential mode hopping or multi-moding problems. This combination of non-planar backward phase-conjugate and forward phasematched geometries produces two DFWM signal beams out of plane away from any of the input beams. Collection of each signal beam can be done without the use of a beam splitter (thereby avoiding losses) and with minimum optical interference. The ICP is mounted between the templates on a 2-D translational stage in order to selectively probe the desired analytical regions of the torch from $3 \mathrm{~mm}$ to $10 \mathrm{~mm}$ above the glass bonnet.

Each signal beam is measured by a simple photodiode (Thorlabs, Inc., model PDA55). To improve signal-to-noise ratio, the forward pump beam is chopped by a mechanical chopper (Stanford Research Systems, Inc., model SR540) and the signal is processed by a lock-in amplifier (Stanford Research Systems, Inc., model SR810DSP). The lock-in output is digitized by a personal computer-based data acquisition system. The perturbation theory program, written in Fortran, is used to fit the measured spectra. Temperature and collisional width are determined from the theoretical fit of the experimental data. Each frequency scan takes approximately 3 minutes to acquire. The probe volume is relatively small $(13 \mu \mathrm{L})$ and the longest dimension is 
oriented along azimuthally, so that the probe volume does not span steep mean temperature gradients.

\section{Results and Discussion}

Many parameters affect the intensity of the wave-mixing signal. For instance, a small difference in RF power creates a hotter or cooler torch, changing the population of the probed state, which changes the signal intensity. From a DFWM signal perspective, the optimal RF range depends on the energy required to disassociate molecules or to populate any non-resonant states. The DFWM signal intensity drops off at RF power levels lower than about $1000 \mathrm{~W}$ due to the lack of energy to sufficiently excite the atoms to this level. An RF power level sufficient to populate the probed state is used in all our experiments in this study.

Inductively coupled plasmas are often used for liquid analytes introduced by a nebulizer. The solution flow introduces deionized water along with the analyte into the torch, adding large, slow molecules to the center region, and in effect, cooling this center part of the torch. However, this is not required for torch operation. Nonetheless it is interesting to know how much the addition of water cools the torch. Figure 3 shows the results of the forward- and backwardgeometry signals taken simultaneously under two different experimental conditions. Figure 3a shows results with no nebulizer gas flow in the central channel while Figure $3 \mathrm{~b}$ shows results with the nebulizer flow on. The measured temperatures are $8,100 \pm 150 \mathrm{~K}$ (without nebulization) and 3,400 $\pm 150 \mathrm{~K}$ (with nebulization). The cooling effect of the nebulizer flow on the plasma torch temperature profile is apparent. 
In order to measure temperature levels in the torch, the diode laser is scanned to obtain spectral profiles for both wave-mixing geometries simultaneously. The spectra are imported into the fitting program described previously and iteratively fitted with temperature and collisional width as variables. Figure 4 shows reproducibility of our experimental data and the line-shape fitting procedures for multiple scans. The data points are connected by straight lines. Figure $4 \mathrm{a}$ shows the forward-geometry results and Figure $4 \mathrm{~b}$ shows the backward-geometry results. The results for fitting each data set are listed in Table 1, along with the residual (chi-squared error). The measured temperatures differ by less than $50 \mathrm{~K}$ (less than $1 \%$ of the measured temperature). In order to test the error in the fitting program based on the initial estimate in temperature, $\mathrm{T} 1$ is varied by up to $5,000 \mathrm{~K}$, while fitting the same set of spectra. When changing this initial estimate, the line shape-fitting procedure yields temperature and line width uncertainties of \pm 10 $\mathrm{K}$ and $\pm 0.0001 \mathrm{~cm}^{-1}$, respectively.

We also consider the effect the sampling rate has on the variability of the measurements provided by the fitting program. By taking the same set of spectra and cutting the effective sampling rate down by one third, it introduces variability in the results of $\pm 150 \mathrm{~K}$ and \pm 0.005 $\mathrm{cm}^{-1}$. The temperature gradients are steep within the torch, which results in a large change in temperature with a small change in the torch position. These deviations in temperature changes result more from changes in experimental parameters than from any potential errors in the fitting program. Hence, we estimate that the overall uncertainty in the measured temperature and line width from a single measurement is approximately $\pm 150 \mathrm{~K}$ and $\pm 0.005 \mathrm{~cm}^{-1}$. This uncertainty in the line width corresponds to an uncertainty in electron number density of $\pm 0.3 \times 10^{15} \mathrm{~cm}^{-3}$. 
To map the torch, lateral measurements of temperature are determined at various torch heights above the bonnet as illustrated in Figures 5 and 6 . Vertical error bars indicate the uncertainty in the measured temperature. Using a standard power level of $1048 \mathrm{~W}$, the maximum temperature is determined to be $14,000 \pm 150 \mathrm{~K}$ at $3 \mathrm{~mm}$ above the bonnet. A good radial profile is obtained at each torch height demonstrating the expected "doughnut" temperature profile of the ICP torch with a hollow colder center.

It was discussed above and also has previously been reported that Stark broadening dominates the homogenous broadening for this transition [19]. Using the homogenous broadening and temperature measured at each spot, one can calculate the electron number density based on Eq. 9. A contour map of the calculated electron densities is shown in Figure 7. The electron density is found to range from $6.1( \pm 0.3) \times 10^{15} \mathrm{~cm}^{-3}$ to $10.1( \pm 0.3) \times 10^{15} \mathrm{~cm}^{-3}$. De Regt et al. [7] reported similar results using a diode laser-based absorption technique.

\section{Conclusions}

In this study, we use a new multi-photon optical setup to collect both the forward- and the backward-geometry wave-mixing spectra simultaneously. The technique is applied to an atmospheric radio-frequency inductively coupled plasma (RF-ICP) torch operating with argon. Inexpensive low-power diode lasers are used to measure an argon transition at $811.754 \mathrm{~nm}$. This non-intrusive technique yields spectra that can be subsequently processed to determine temperature and homogeneous broadening values. Temperatures are measured vertically and horizontally across the radius of the torch. The temperature measurements result in a range of 3,500 to $14,000 \mathrm{~K} \pm 150 \mathrm{~K}$. The uncertainty is found to be less then $5 \%$. Electron densities measured range from $6.1( \pm 0.3) \times 10^{15} \mathrm{~cm}^{-3}$ to $10.1( \pm 0.3) \times 10^{15} \mathrm{~cm}^{-3}$. 


\section{Acknowledgments}

We gratefully acknowledge partial support of this work from the National Institute of

General Medical Sciences, National Institutes of Health under Grant No. 5-R01-GM41032, and Varian, Inc. 


\section{References}

1. V. Donney, M. Schabel: Journal of Applied Physics 91, 6288 (2002)

2. T. Owano, T. Gordon, C. Kruger: Phys. Fluids B 2, 3184 (1990)

3. D. Baer, R. Hanson: J. Quant. Spectrosc. Radiat. Transfer 47, 455 (1992)

4. J. De Regt, R, Tas, J. van de Mullen: J. Phys. D: Appl. Phys. 9, 2404 (1996)

5. C. Wang, F. Mazzotti, P. Miller, C. Winstead: Appl. Spec. 56, 386 (2002)

6. D. Baer, A. Chang, R. Hanson: J. Opt. Soc. Am. B 9, 1968 (1992)

7. J. De Regt, F. de Groote, J. van der Mullen, D. Schram: Spectrochimica Acta Part B 51, $1371(1996)$

8. A. Eckbreth: Laser Diagnostics for Combustion Temperature and Species (Second Edition) (Gordon and Breach Publishers, Amsterdam, The Netherlands 1996).

9. P. Danehy, E. Friedman-Hill, R. Lucht, R. Farrow: Appl. Phys 57, 243 (1993)

10. D. Green, T. Owano, S. William, D. Goodwin, R. Zare, C. Kruger: Science 259, 1726-4 (1993)

11. K. Musiol, K. Dzierziga, E. Pawelec, B. Pokrzywka, S. Pellerin, S. Labuz: J. Phys. D: Appl. Phys. 30, 2234 (1997)

12. R. Fisher, Optical Phase Conjugation (Academic Press, New York, 1983) 
13. P. Danehy, R. Farrow and R. Lucht: Proceedings of The First Australian Conference on Laser Diagnostics in Fluid Mechanics and Combustion, Sydney, 95 (1996)

14. P. Danehy, R. Farrow, R. Lucht, T. Reichardt: Proceedings of The Pacific Rim Conference on Lasers and Electro-Optics, Seoul Korea, The Institute of Electrical and Electronics Engineers, Piscataway, NJ, 756 (1999)

15. J. Nilsen, A. Yariv,: J. Opt. Soc. Am. 71, 180 (1981)

16. R. Abrams, R. Lind: Opt. Lett. 2, 94 (1978)

17. R. Abrams, J. Lam, D. Lind, D. Steel, P.Liao: Optical Phase Conjugation (Academic Press, New York, New York 1983)

18. P. Danehy: Population- and thermal-grating contributions to degenerate four-wave mixing, Ph.D. dissertation (Stanford University, Stanford, CA 1995)

19. D. Baer: HTGL report no. $t-286$ (Stanford University, Stanford, CA 1993)

20. H. Griem: Spectral Line Broadening by Plasmas (Academic Press, New York, NY 1974) 


\section{Figure Captions}

Figure1. Schematic diagram of the plasma torch and relative positions of the regions probed. The origin of the coordinates is shown at the top of the glass bonnet above the nebulizer.

Figure 2. Experimental setup for dual forward-and-backward wave mixing.

Figure 3. Forward and backward signals are taken simultaneously with (a) no nebulizer gas flow and no water introduced into the torch. The measured temperature is $8,100 \mathrm{~K}$. The measured temperature is $3,400 \mathrm{~K}$ (b) when the nebulizer flow is on and deionized water is introduced into the torch.

Figure 4. Reproducibility results for three scans collected for (a) forward geometry peaks and (b) backward geometry peaks.

Figure 5. Plot of measured temperatures across the radius of the torch using $1.25 \mathrm{~mm}$ increments from the center and $3 \mathrm{~mm}$ and $5 \mathrm{~mm}$ above the bonnet.

Figure 6. Plot of calculated temperatures across the radius of the torch at $8 \mathrm{~mm}$ and $10 \mathrm{~mm}$ above the bonnet.

Figure 7. Contour plot of the electron density (given in units of $10^{15} \mathrm{~cm}^{-3}$ ) in the analytical zone of the torch. 
Table 1: Results obtained from dual forward-and-backward wave-mixing setup.

\begin{tabular}{|l|l|l|l|l|}
\hline Run & $\begin{array}{l}\text { Temperature } \\
\text { measured }\end{array}$ & $\begin{array}{l}\text { Collision width } \\
\text { measured }\end{array}$ & $\begin{array}{l}\text { Residual } \\
\text { (Chi Sqr) } \\
\text { Forward }\end{array}$ & $\begin{array}{l}\text { Residual } \\
\text { (Chi Sqr) } \\
\text { Backward }\end{array}$ \\
\hline 1 & 8618.4 & 0.10608 & $2.3 \times 10^{-3}$ & $3.1 \times 10^{-3}$ \\
\hline 2 & 8567.4 & 0.10966 & $4.0 \times 10^{-3}$ & $2.3 \times 10^{-3}$ \\
\hline 3 & 8599.4 & 0.10790 & $3.1 \times 10^{-3}$ & $3.0 \times 10^{-3}$ \\
\hline
\end{tabular}




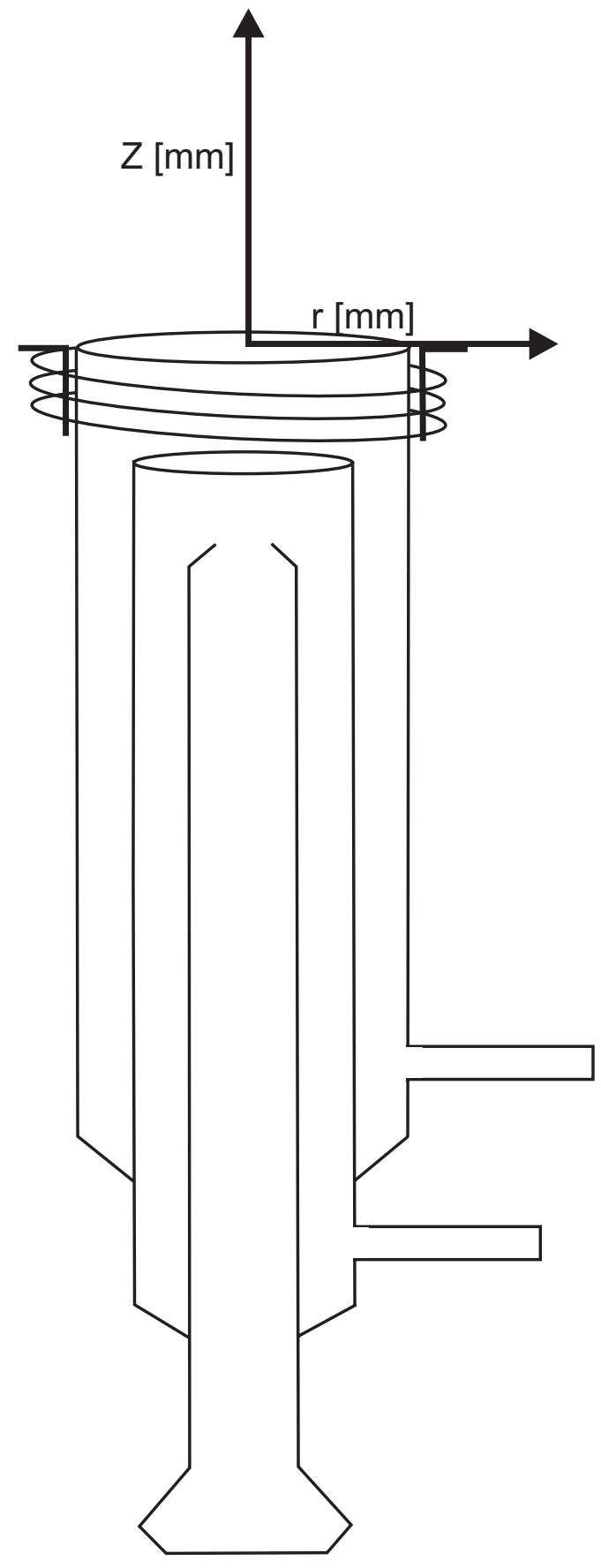

Figure 1 


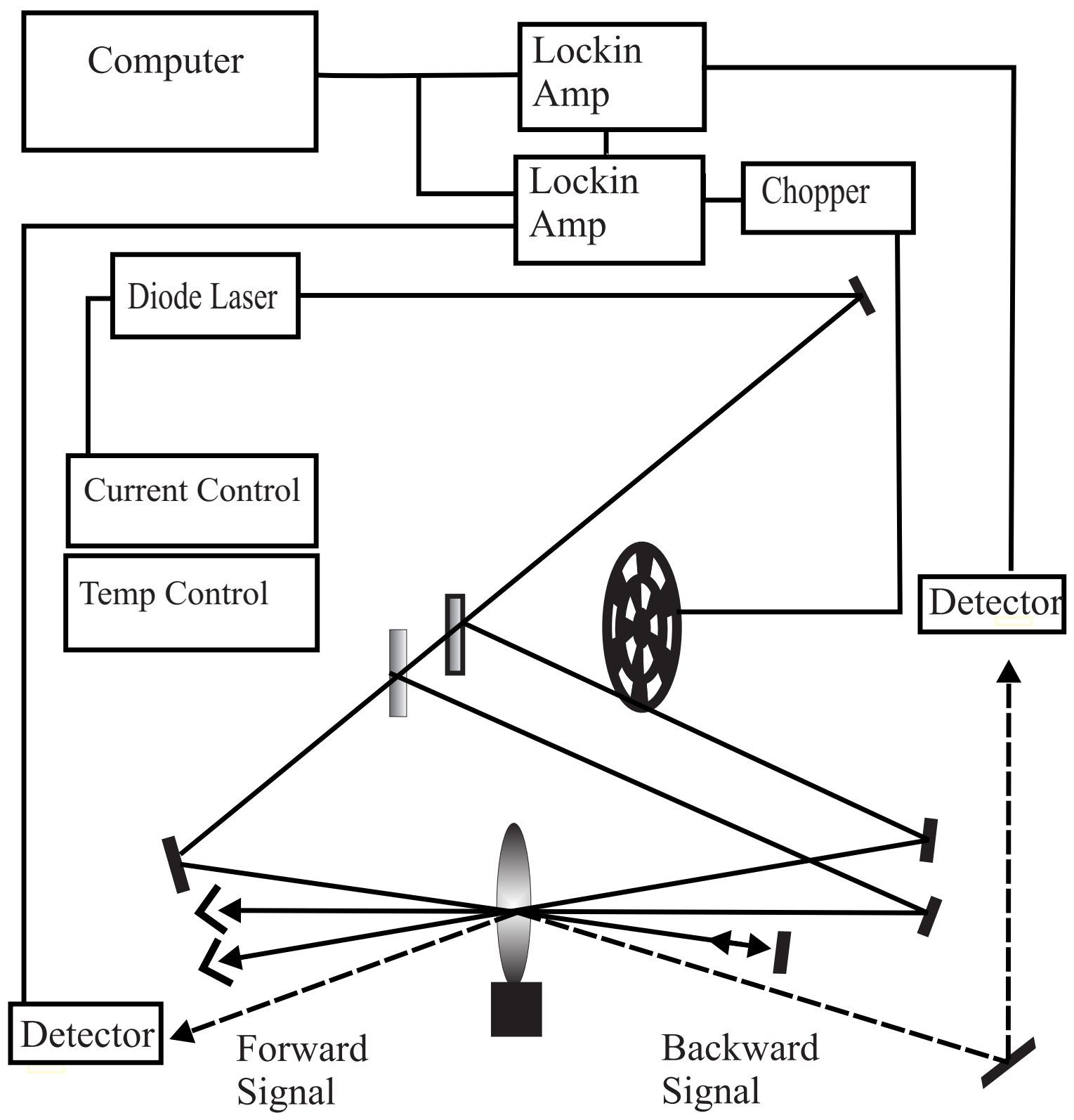

Figure 2 


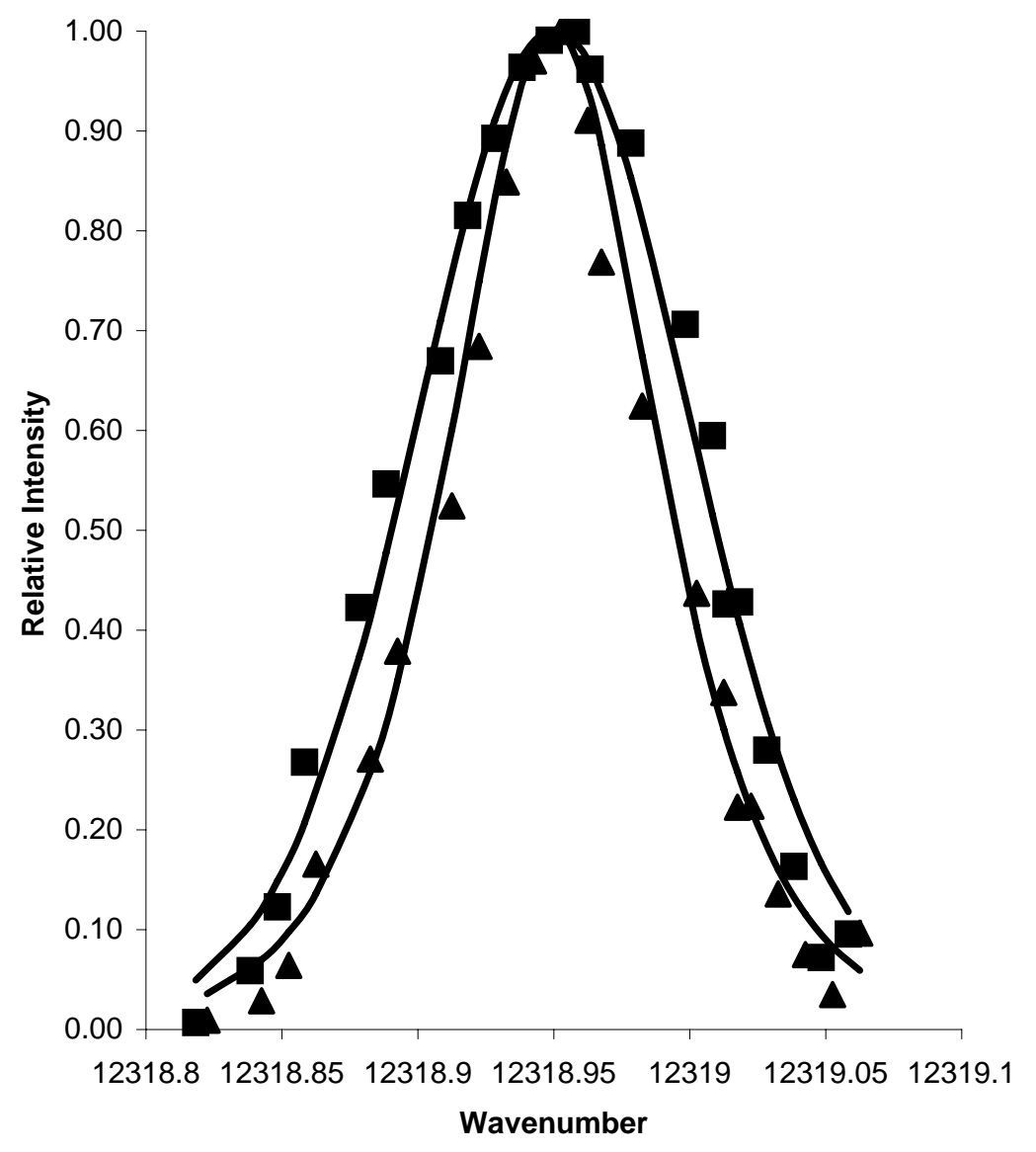

Figure 3(a) 


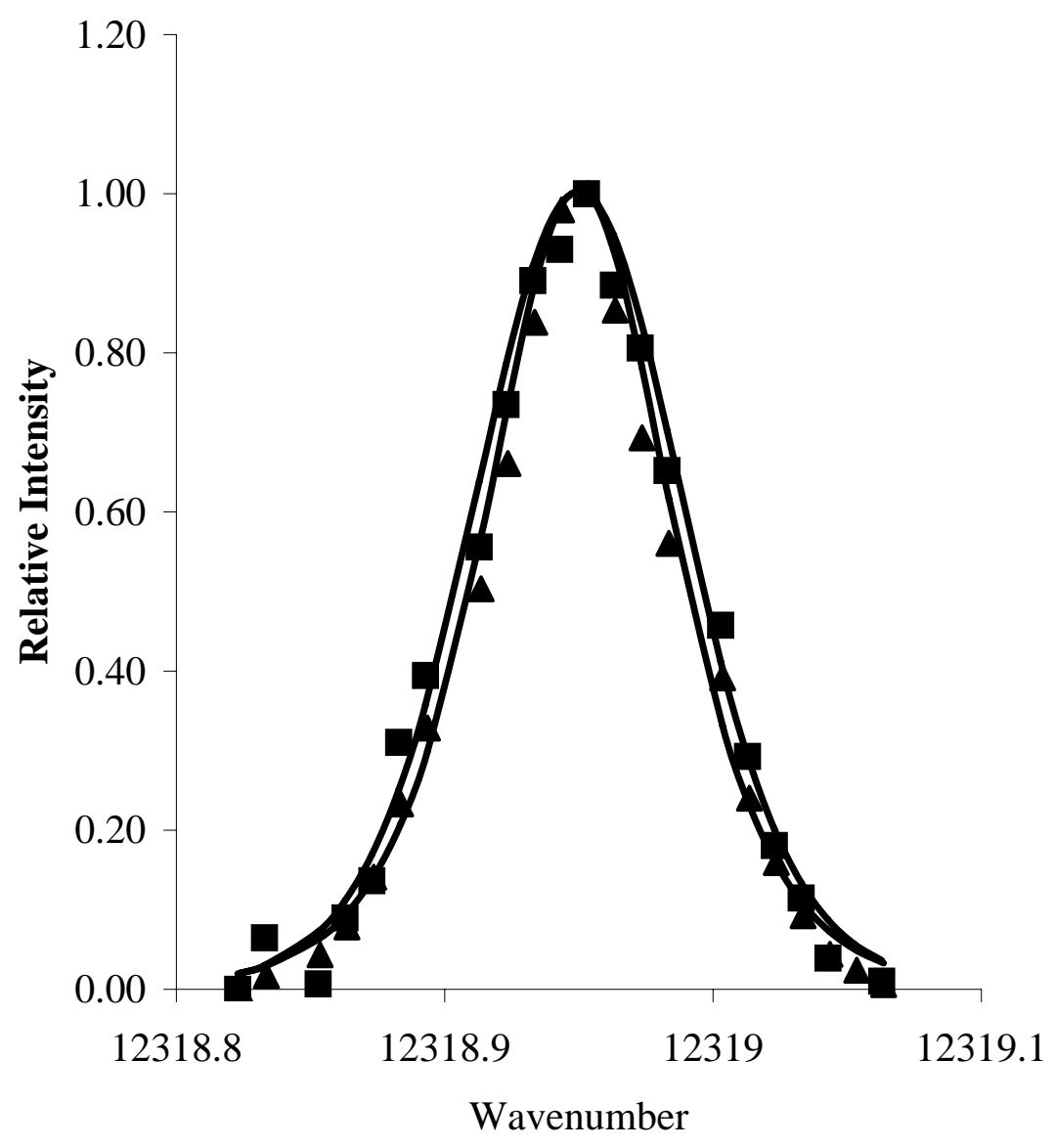

Figure 3(b) 


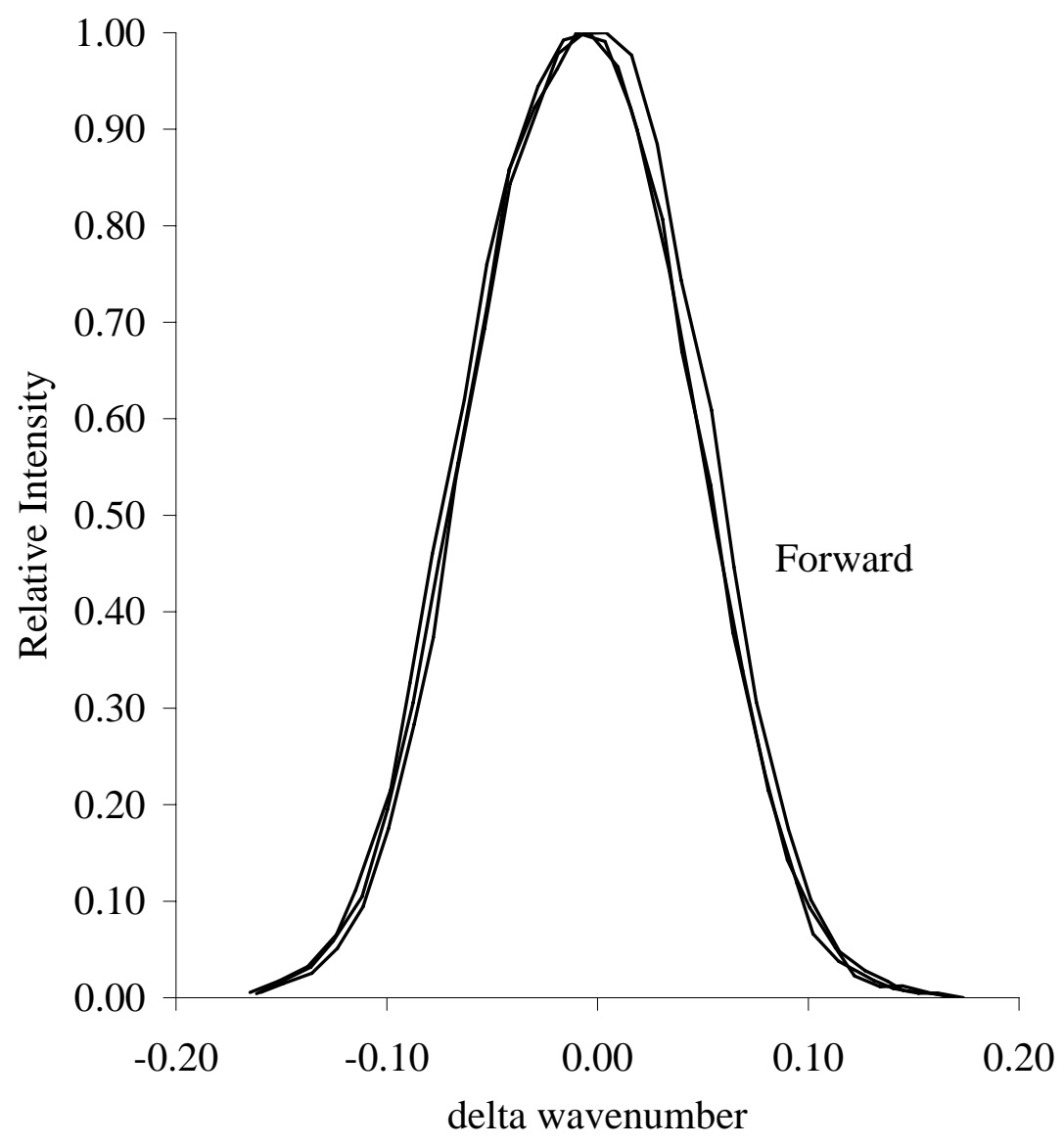

Figure 4(a) 


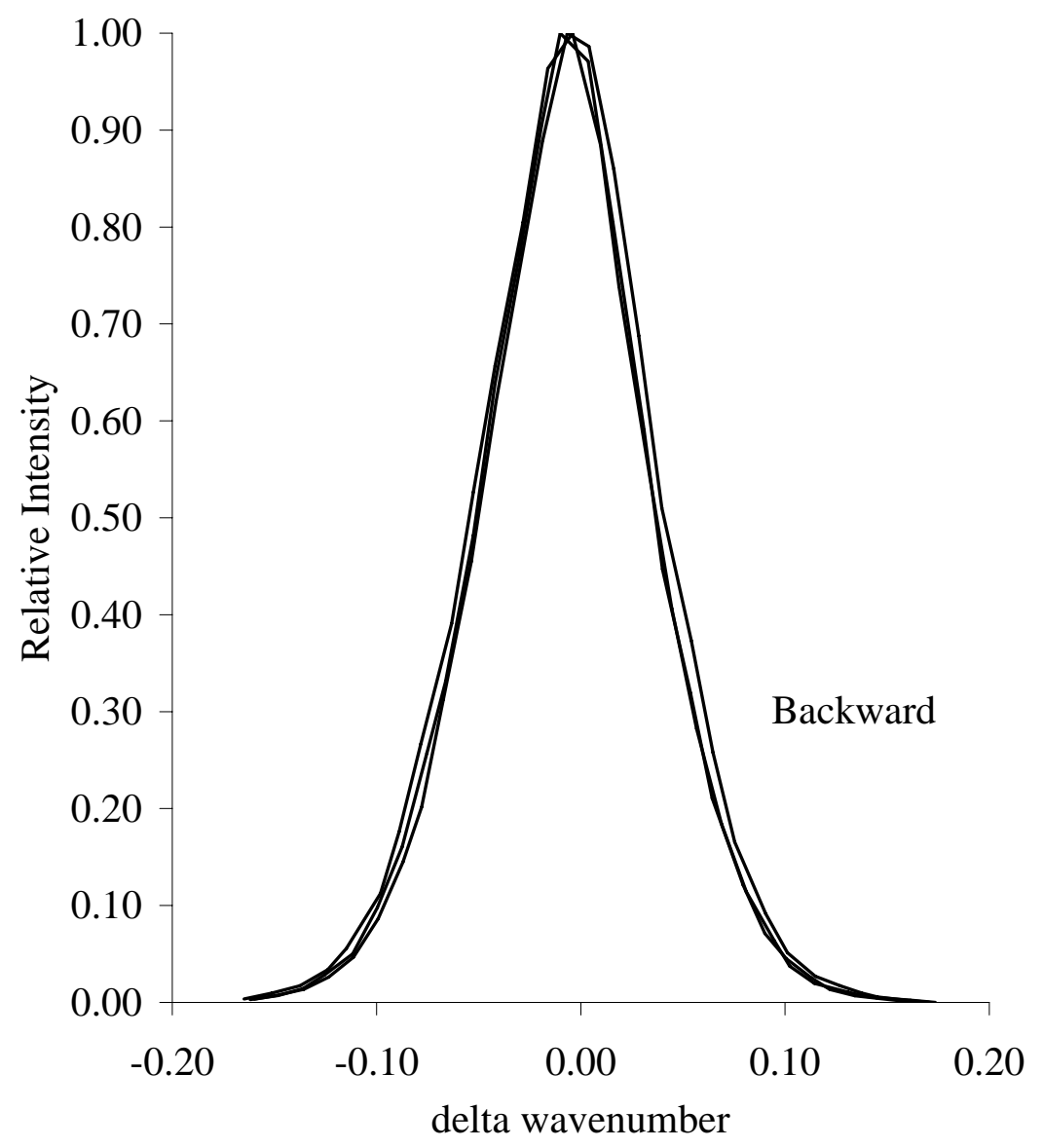

Figure 4(b) 


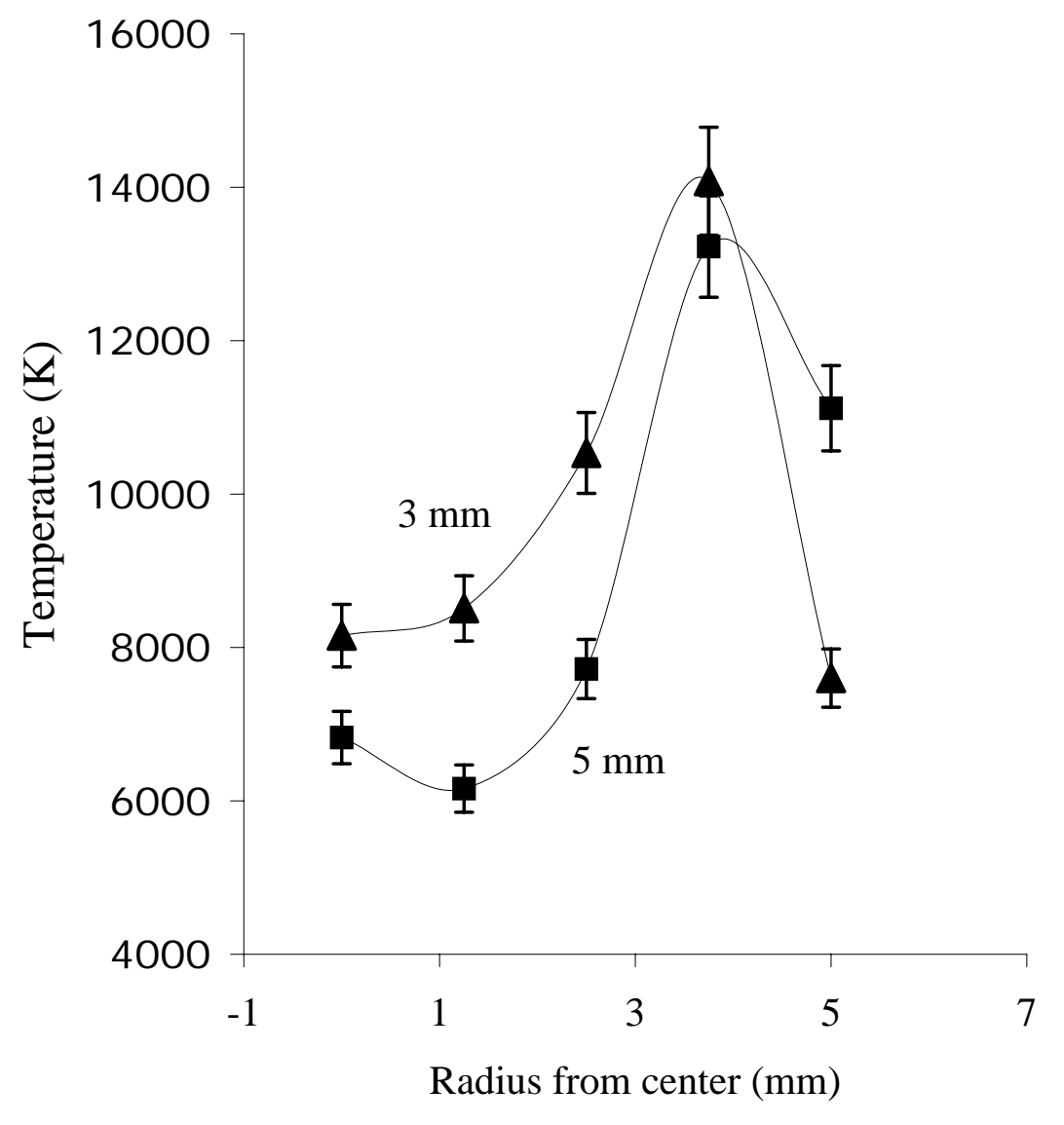

Figure 5 


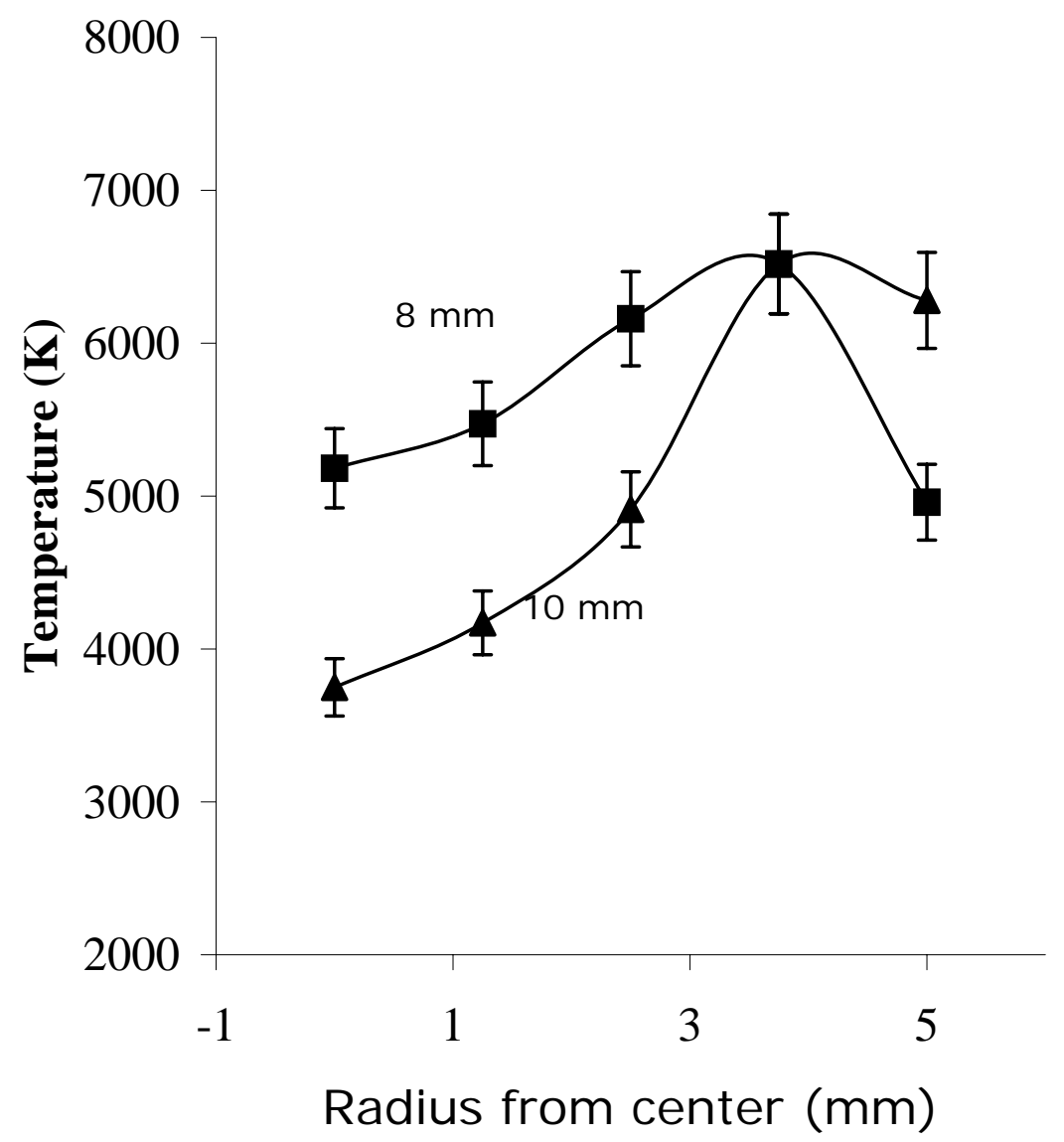

Figure 6 


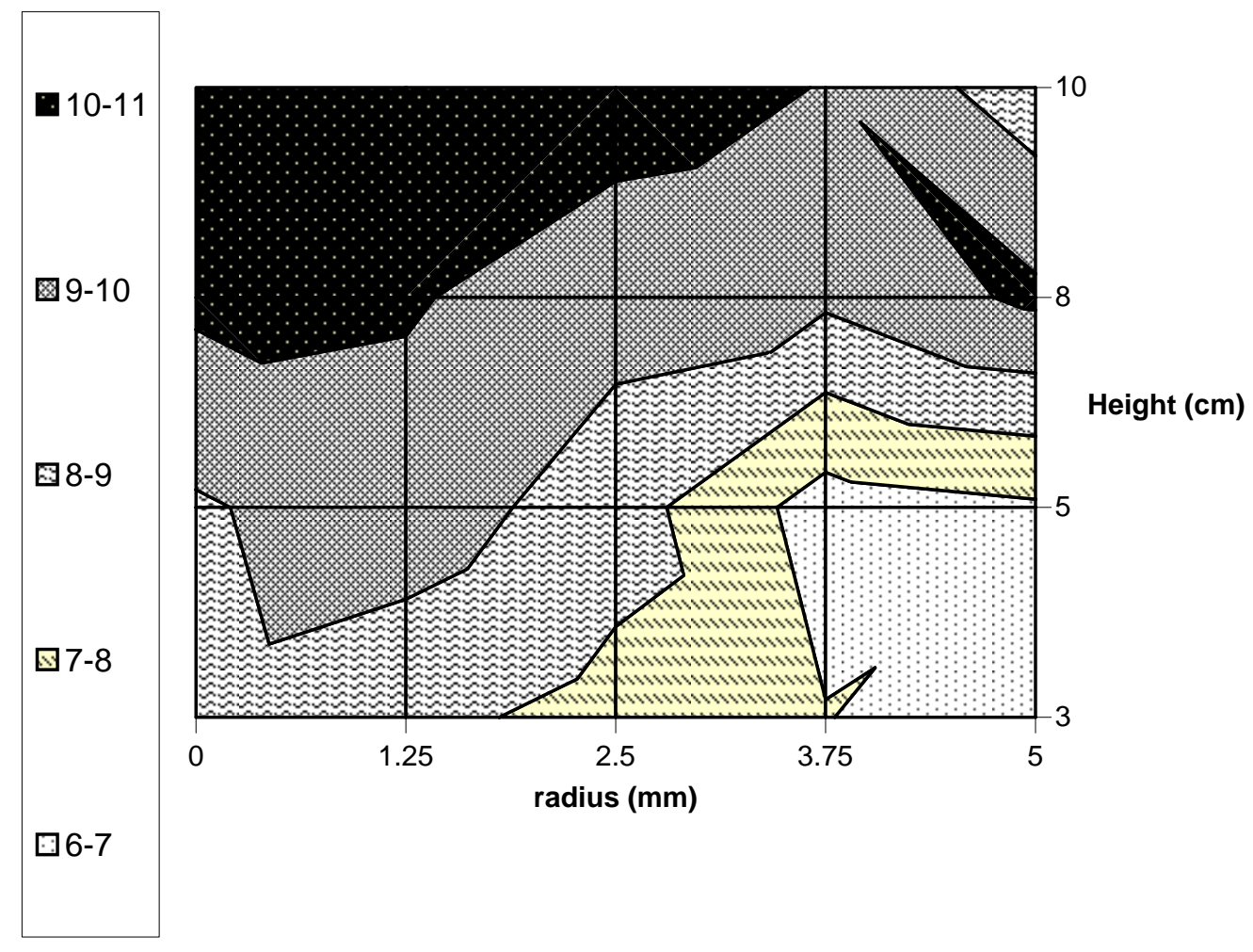

Figure 7 